\title{
CÁlCULO DE ESTRUCTURAS DE FÁBRICA ARMADA CONTRA EL SEISMO
}

\author{
(REINFORCEMENT IN SEISMIC DESING OF MASONRY STRUCTURES)
}

G. Michele Calvi, Profesor Dr. Ingeniero

Dipartamento di Meccanica Strutturale Università degli Studi di Pavia.

ITALIA

Fecha de recepción: 20-VI-92

$631-11$

\section{RESUMEN}

Se exponen las consideraciones básicas a tener en cuenta al calcular "edificios sencillos" construidos con obras de fábrica, que puedan verse sometidos a vibraciones sísmicas.

También se analiza la influencia que, frente al sismo, tienen las tan habituales "fábricas confinadas", es decir, las estructuras de pórticos reticulares con plementería de fábrica rellenando los pórticos.

Tras el análisis de la casuística de fallo de los edificios de fábrica, sometidos al sismo, se orienta sobre la disposición de las armaduras que pueden paliar los efectos sísmicos.
SUMMARY

The article deals with the basic considerations to be kept in mind when designing "simple buildings" built with masonries which can be exposed to seismic vibrations.

The influence of so usual "confined masonries" on the seismic wave is also analyzed. These masonries are actually reticulate frames with masonry panels filling them.

After analyzing mechanisms of failures in masonry buildings exposed to earthquake, the article concentrates on the layout of reinforcements which can diminish seismic effects.

\section{INTRODUCCIÓN}

Este documento es, esencialmente, una colección de ideas que sirvan de hilo conductor del tema de cuándo y cómo es conveniente armar contra la sismicidad las estructuras de obra de fábrica, ya tenga dicha fábrica función de elemento carga o de plementería no portante.

Existen algunos documentos de normativa, como las Recomendaciones $\mathrm{CIB}^{(1)}$, el Eurocódigo ${ }^{(2)}$, el Eurocódigo $6^{(3)}$, y algunos códigos nacionales, que son bastante pobres en casi todos los casos con respecto al estado actual de los conocimientos técnicos sobre la materia.

A nivel científico la literatura es amplia, pero por desgracia los distintos tipos de materiales estudiados bajo la muy genérica definición de obra de fábrica son también muy numerosos. Los números (5), (6) y (7) de la bibliografía son sólo ejemplos de los textos más destacados que se han editado.

\section{CONCEPTOS BÁSICOS}

En una primera ojeada, hay tres clases de materiales que nos interesan:

- ladrillos cerámicos macizos,

- ladrillos cerámicos huecos,

- bloques de hormigón hueco. 
El posible objeto de la armadura depende de la calidad y de la función del elemento de fábrica y puede describirse grosso modo como sigue:

- aumentar la resistencia,

- aumentar la ductilidad,

- evitar un colapso localizado.

El primer objetivo suele requerir un alto porcentaje de armadura y se aplica fundamentalmente a los bloques de hormigón, para los que la filosofía de cálculo se resume en igualar lo más posible su cálculo al comportamiento de una estructura de hormigón bien proyectada.

El segundo y tercer objetivo se alcanza, en casi todos los casos, con una baja proporción de armadura y se aplica a todas las clases de muros de fábrica, tabiques y plementería de fábrica.

El efecto de una estructura de fábrica con armadura repartida se describe claramente en C.6.3.3.3. del EC8:

"La armadura repartida evita el colapso localizado después del agrietamiento y mantiene la integridad del muro en el estado plástico. Mejora la ductilidad existente y reduce la posibilidad de deterioro.

Por lo general, con la armadura repartida no se persigue aumentar la resistencia al cizallamiento respecto a la obra sin armar, sino reducir el deterioro y pérdida de rigidez, permitiendo mayores deformaciones plásticas.

La armadura mínima especificada debe asegurar que el fallo tensional de las juntas no ocasione una rotura frágil del paramento.

Debe señalarse también que la presencia de excesivo armado horizontal puede dar lugar a roturas por compresión de las piezas antes de que el acero entre en período plástico, lo que supondría un comportamiento frágil a rotura".

\section{PLANOS DE OBRA}

La elaboración de buenos planos de detalle constructivos es esencial para evitar el colapso localizado y para aumentar la ductilidad general; es menos importante en cuanto al aumento de la resistencia de la estructura. En el estado actual del cálculo antisísmico está bien establecido que una de las exigencias determinantes consis- te en que la estructura sea relativamente insensible a un tipo de excitación (aceleración punta y frecuencia presente), y a la respuesta real en el estado no lineal (el comportamiento previsto en el análisis puede ser bien distinto de la respuesta real). Este objetivo se puede buscar proyectando los detalles con todo cuidado, considerando la estabilidad de la respuesta $o$, dicho de otro modo, con un grado de ductilidad fiable no afectado por algún fallo prematuro localizado.

Las cláusulas 6.3.3., 6.4.1. y 6.4.2. del EC8 indican ciertas reglas básicas que deben cumplirse; algunos requisitos parecen referidos al buen proyecto de un edificio sismorresistente (p. ej.: las cifras deben considerarse como sugerencias, que los estados miembros fijarán con mayor exactitud):

- la distancia vertical entre armaduras horizontales no debe exceder de $600 \mathrm{~mm}$;

- deben ponerse armaduras de acero de diámetro no inferior a $4 \mathrm{~mm}$ dobladas alrededor de barras verticales en los extremos del muro;

- el porcentaje mínimo de armadura horizontal repartida en el muro, respecto a la sección del mismo, no debe ser inferior a $0,5 / 1000$;

- deben disponerse barras verticales de sección no inferior a $4 \mathrm{~cm}^{2}$ en ambos extremos libres de todos los muros, en todos los encuentros, y dentro del muro mismo, siempre que sea preciso, para no exceder el intervalo de $4 \mathrm{~m}$,

- el reparto mínimo de armado en el muro, referido a la sección del mismo, no debe ser inferior a $0,5 / 1000$

- el espesor del muro no debe ser inferior a $240 \mathrm{~mm}$;

- la relación entre la altura libre y el espesor del muro no debe ser mayor de 15.

También se exige explícitamente que el comportamiento de los muros y forjados de la estructura sea tridimensional, contribuyendo todos los elementos a la resistencia de la carga aplicada; por lo tanto deben disponerse muros de arriostramiento ortogonales en dos sentidos y debe verificarse que los forjados puedan transmitir los empujes horizontales a los muros.

También conviene fijar reglas particulares expresadas para definir el concepto de "edificio sencillo", para el que las verificaciones estructurales (comentadas posteriormen- 
te) se reducen a una revisión implícita de la sección resistente de los muros, más algunas reglas consideradas satisfactorias.

Se acepta esta simplificación porque la experiencia demuestra que los edificios de fábrica bien proyectados y construidos se comportan bien en condiciones sísmicas extremas en zonas de alta sismicidad.

Un "edificio sencillo" debe cumplir todas las prescripciones aplicables a un edificio normal, más las que se resumen a continuación:

- Las plantas se limitan a un número variable entre 3 y 5 , según la aceleración punta del suelo considerada en el cálculo.

- La forma de la planta debe ser aproximadamente rectangular, con una relación entre lados no superior a 0,25 , y con entrantes y salientes del plano rectangular no mayores del $15 \%$ respecto al lado paralelo a los mismos, y con un máximo de $2 \mathrm{~m}$.

- Por lo menos el $75 \%$ de la carga vertical debe recaer sobre los muros.

- La distribución de los principales muros de carga interiores ha de ser simétrica respecto a dos ejes ortogonales.

- Deben situarse dos muros paralelos de longitud no inferior al 50\% de la del edificio en el sentido de dichos muros, separados entre sí a más del $75 \%$ de las otras dimensiones en planta del edificio.

- La diferencia de la masa de la sección horizontal de los muros de un piso a otro no debe exceder del $20 \%$.

- La sección horizontal de los muros resistentes al empuje lateral, en ambos sentidos ortogonales, expresada en porcentaje del área de las plantas por encima del nivel considerado, no debe ser menor en ninguna planta a 2, 4 ó 5, según sea la aceleración punta del suelo considerada en el proyecto.

\section{ANÁLISIS Y CÁLCULO}

El cálculo de estructuras de fábrica armada, como la de cualquier estructura antisísmica, exige definir el estado límite que debe evitarse, el grado fiable de ductilidad que aporte la estructura, el método de análisis apropiado al caso y la verificación de seguridad que deba efectuarse.
Es común a efectos prácticos agrupar todos los fenómenos no lineales que contribuyen a disipar la energía en un solo factor reductor de esfuerzos (también denominado factor de comportamiento o factor q) que esta, por tanto, en principio, en función del estado límite de proyecto y de la ductilidad disponible.

De este modo es posible efectuar los análisis lineales con un grado idóneo de carga aplicada, que corresponde a la carga prevista en el caso de respuesta lineal reducida por el factor $q$.

El EC8 (C1. 6.5.) recomienda tomar un factor q de 2,5 para las estructuras de fábrica armada, unido a un coeficiente de amortiguamiento igual al $\mathbf{8 \%}$ del crítico.

Se considera apropiado para los edificios de obra de fábrica un análisis dinámico simplificado $(\mathrm{Cl}$. 5.3.1.2. de la parte 1.1). Puede realizarse generalmente un análisis lineal asimilando el edificio completo y considerando los forjados rígidos en su propio plano.

Si el modelo estructural tiene en cuenta la conexión entre las vigas, puede hacerse un análisis de la estructura para determinar los efectos de la acción sobre los elementos horizontales y verticales de ella ( $\mathrm{Cl}$. 6.6.2.).

El análisis dinámico simplificado, anteriormente citado, implica evaluar el modo fundamental de vibración y aplicar al modelo una serie de esfuerzos horizontales calculados con arreglo al valor de la ordenada del espectro de respuesta correspondiente al período fundamental de vibración.

También se tiene en cuenta implícitamente la respuesta no lineal de la estructura cuando se acepta que puede variarse la distribución de la totalidad del efecto cortante básico entre los muros que resulte del análisis lineal, siempre que se asegure el equilibrio general y que la acción de cualquier muro no se reduzca en más del $30 \%$ ni se incremente en más del $50 \%$.

Para tener un grado de seguridad frente al colapso, habrá que evaluar la resistencia de cálculo de cada elemento antisísmico con arreglo al EC8, considerando el valor apropiado de todos los factores de seguridad (CL. 6.6.3. y 6.1.3).

Puesto que todos los muros están expuestos a esfuerzos horizontales y verticales, tienen importancia tanto las 
prescripciones para muros de arriostramiento como las relativas a los muros portantes de cargas verticales.

Los posibles modos de colapso son bien conocidos y sólo se resumen para mayor claridad:

Respecto al efecto cortante:

- deslizamiento relativo entre juntas horizontales;

- apertura de figuras diagonales en las juntas;

- fisuración diagonal de las piezas;

- fisuración diagonal por compresión de las piezas.

Respecto a la flexión:

- aparición de fisuras horizontales;

- aplastamiento en la base comprimida;

- desplome.

\section{LA ESTRUCTURA CON OBRA DE FÁBRICA CONFINADA}

Las estructuras porticadas reticulares plementadas con muros, tabicones o con particiones de fábrica, merecen especial atención por dos grandes razones:

- es un método de construcción muy común y por ello es alto el porcentaje de edificios de este tipo;

- anteriormente los científicos han prestado muy poca atención a esta clase de edificios, por lo que se nota la necesidad de investigación experimental y matemática.

Se reconoce que la presencia de rellenos de albañilería modifica el comportamiento fundamental de la estructura como pórtico, al rigidizarlo y crear nuevos mecanismos de posible rotura. Por ejemplo, si sólo se rellenan algunos paños de la estructura reticular de un edificio, se rigidizan y polarizan una absorción no homogénea de la acción sísmica; el período natural total de vibración se reduce y el centro de torsiones se puede desplazar, induciendo a un alto riesgo de torsión.

El cálculo de la obra de fábrica confinada exige examinar los posibles mecanismos de colapso que podrían provocar la fractura del relleno (véase también el n. 8 de la bibliografía):

- Colapso por compresión diagonal del arriostramiento.

El colapso por compresión del arriostramiento no tiene relación con la presencia de armadura, y depen-

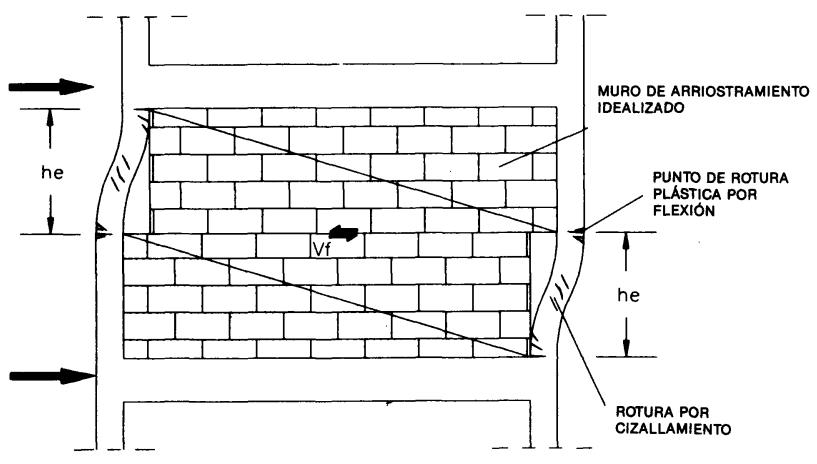

Rotura a cizallamiento por rasante de una fábrica confinada.

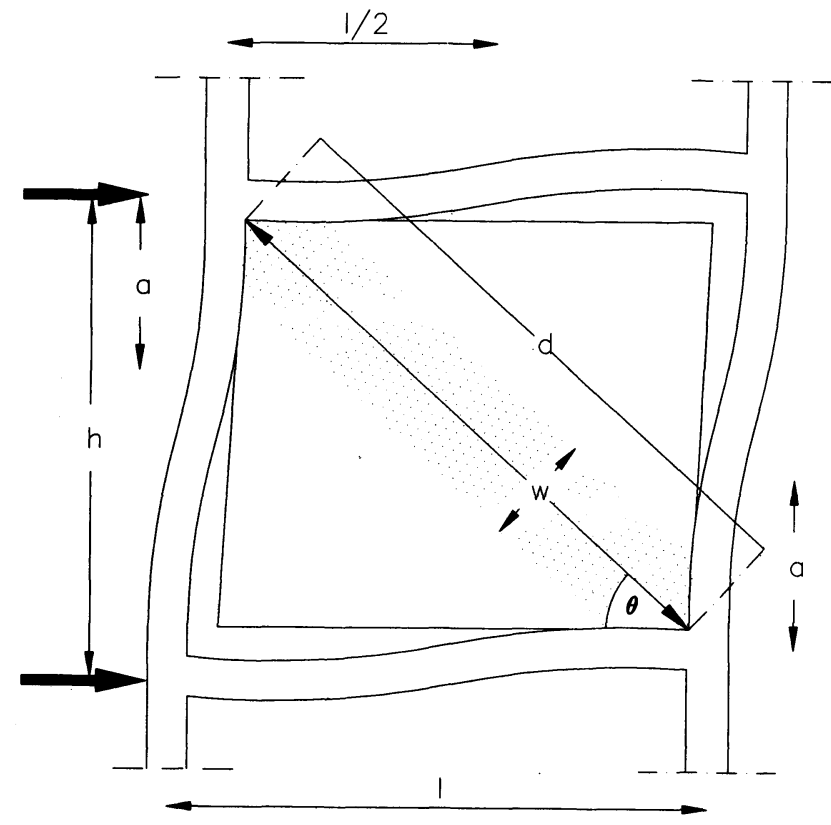

Equivalencia a efectos de arriostramiento de una fábrica confinada.

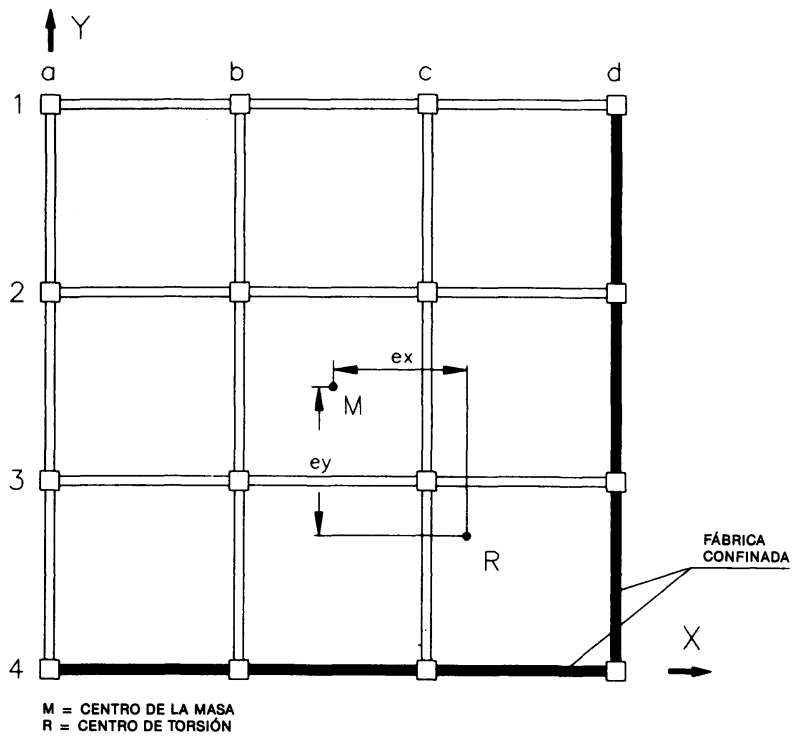

Planta de un edificio de hormigón armado con fábrica confinada de relleno en dos fachadas. 

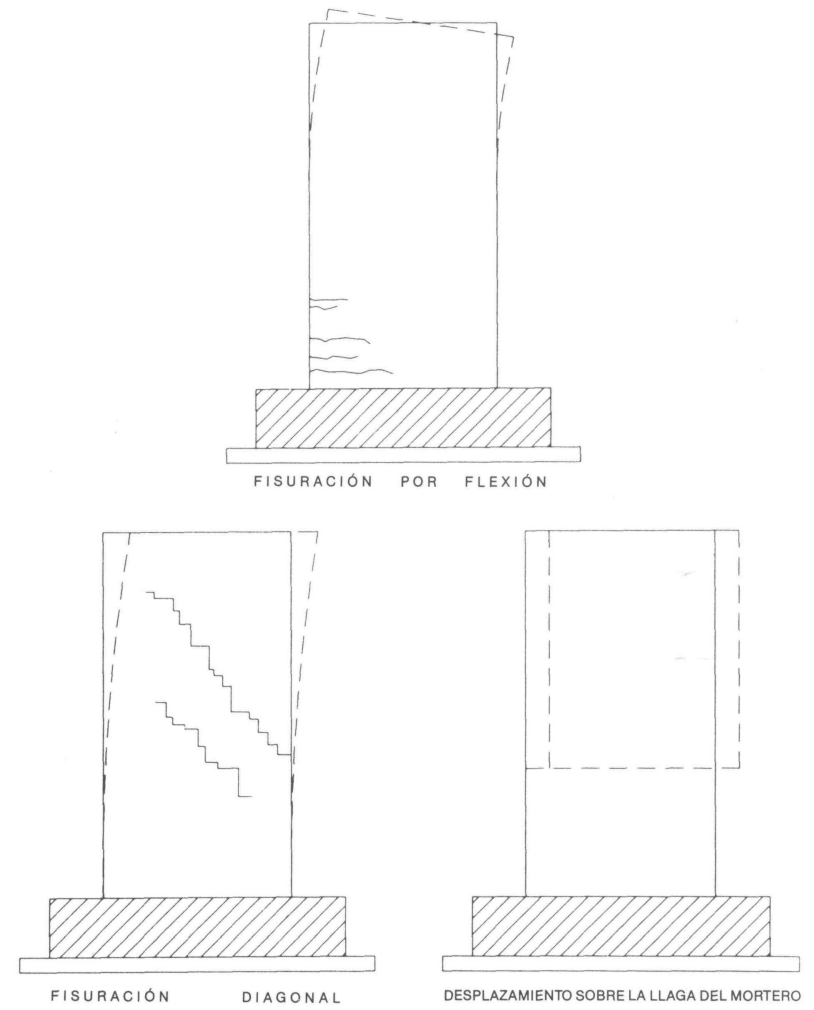

Correlación entre tipos de deformación y su patología.

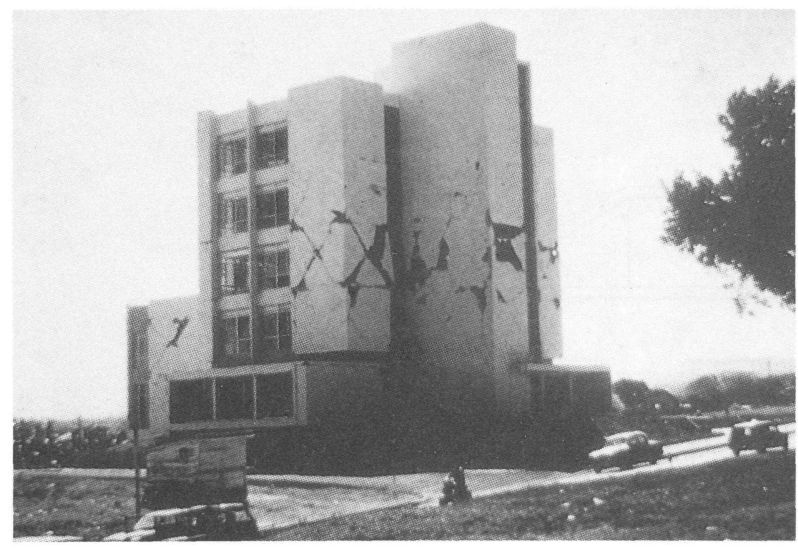

Ejemplo de patología sísmica en un edificio de fábrica.

de esencialmente de la resistencia de la obra de fábrica (considerando el sentido más débil, es decir, perpendicular a los huecos de los bloques o ladrillos) y de la rigidez relativa del relleno y del pórtico.

La tensión de rotura por compresión diagonal se calcula como sigue:

$$
\mathrm{R}_{\mathrm{c}}=\frac{2}{3} \mathrm{ztf}_{\mathrm{m}} \sec \theta
$$

Donde $\mathrm{z}$ es la longitud de contacto entre el paramento y el pilar obtenida mediante:
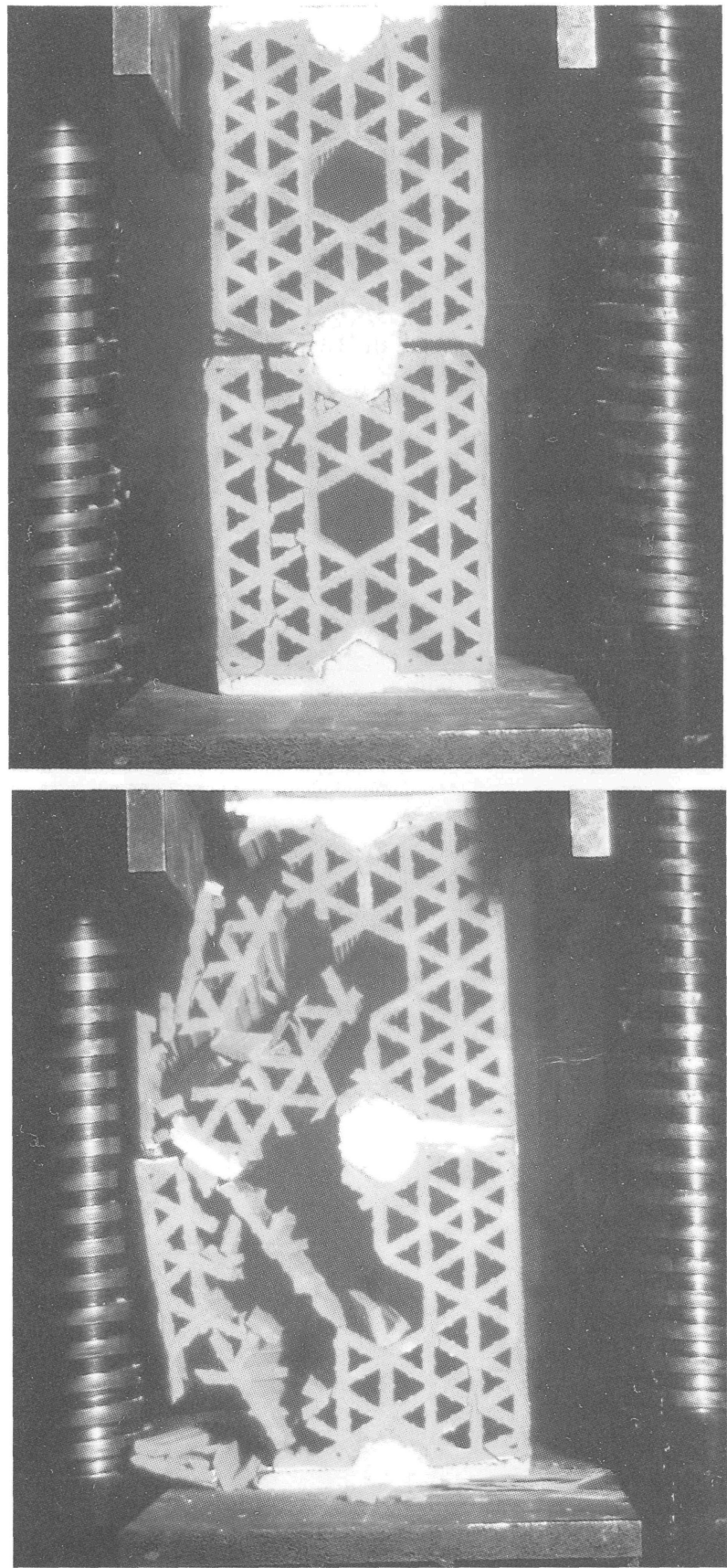

Comportamiento frágil típico de bloques perforados.

$$
\mathrm{Z}=\frac{\pi}{2} \cdot\left[\frac{4 \mathrm{E}_{\mathrm{c}} \mathrm{g} \mathrm{h}}{\mathrm{E}_{\mathrm{m}} \mathrm{t} \operatorname{sen} 2 \theta}\right]^{1 / 4}
$$

$\mathrm{y} t$ es el espesor del muro, $\mathrm{f}_{\mathrm{m}}$ es la resistencia a la compresión de la fábrica, $\mathrm{E}_{\mathrm{c}}$ y $\mathrm{E}_{\mathrm{m}}$ es el módulo de elasticidad del hormigón y del relleno, $\mathrm{I}_{\mathrm{g}}$ es el momento de inercia del pilar de hormigón y $\theta$ es el ángulo de la riostra respecto a la horizontal. Por tanto, la fueza horizontal necesaria para provocar la rotura por compresión es:

$$
\mathrm{V}_{\mathrm{c}}=\mathrm{R}_{\mathrm{c}} \cos \theta
$$



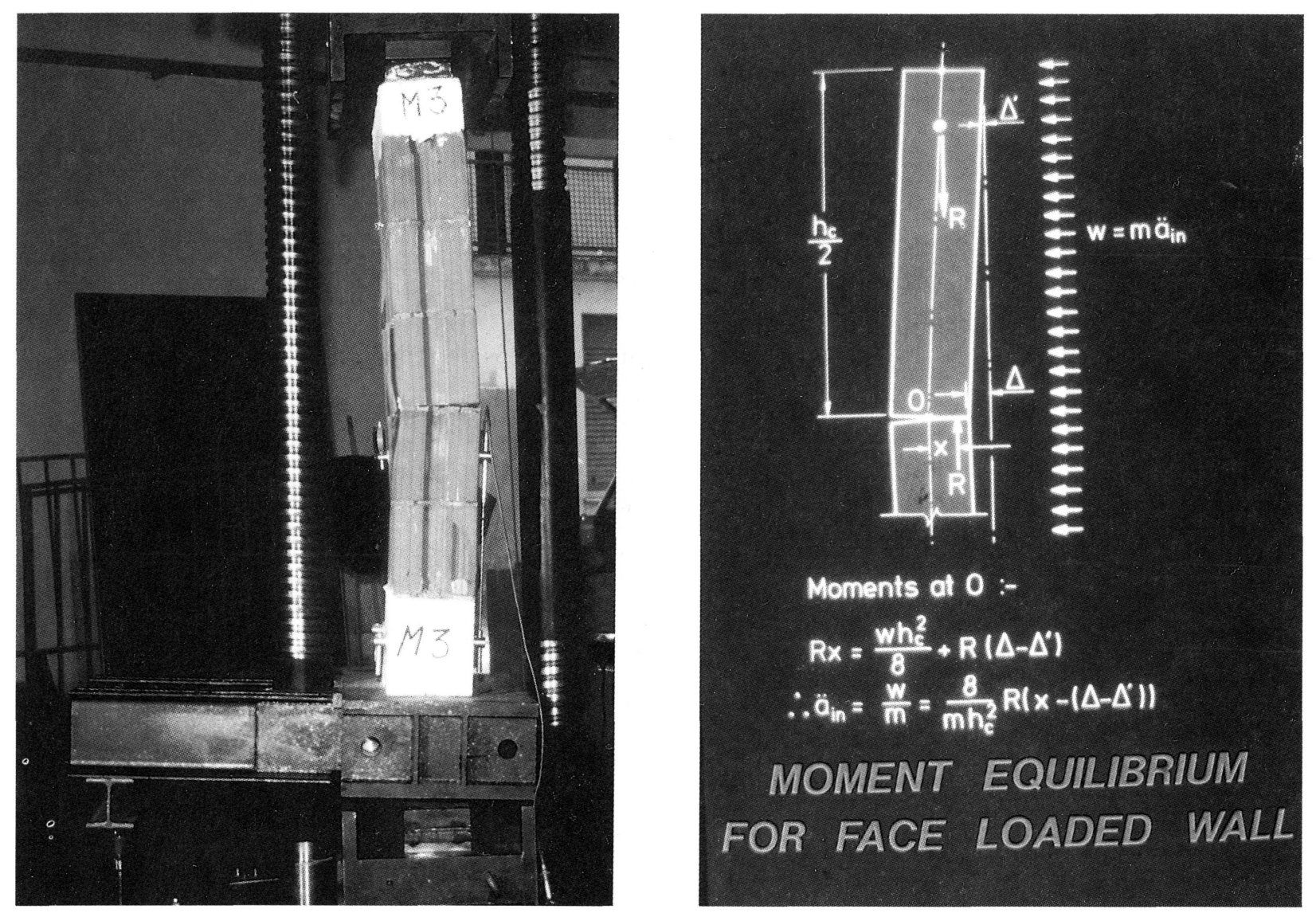

Desplome por distintas solicitaciones.

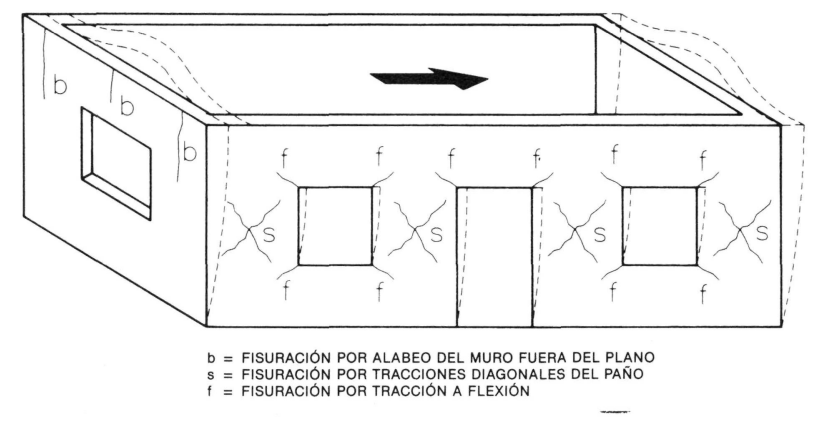

Casuística de un edificio de obra de fábrica sometido al seísmo.

- Fallo por deslizamiento a cortante siguiendo la llaga horizontal del mortero.

Esta forma de colapso depende fundamentalmente del rozamiento entre los ladrillos y el mortero e influye mucho en ella la presencia de armaduras verticales. El esfuerzo de corte, al iniciarse el deslizamiento, se puede calcular a partir de unas cuantas hipótesis (por lo general conservadoras):

- el deslizamiento por cizalladura se produce aproximadamente a media altura del paramento;
- la resistencia a cortante para que inicie el deslizamiento es el 3\% de la resistencia a la compresión;

- la fuerza vertical del paramento sólo procede de la componente de la fuerza de compresión diagonal;

Con un pórticor de $\mathrm{n}$ crujías de aproximadamente la misma longitud 1, el esfuerzo cortante necesario para que se inicie el deslizamiento se puede calcular mediante:

$$
\mathrm{V}_{\mathrm{s}}=\frac{0,03 \mathrm{nf}_{\mathrm{m}}}{1-0,3_{1}^{\mathrm{h}}} \mathrm{lt}+\mathrm{Av}_{\mathrm{v}} \mathrm{f}_{\mathrm{y}}
$$


- Fisuración por tracción diagonal.

Esta forma de rotura no produce el colapso de por sí, pero suele venir seguida por un abombamiento del paramento cuando hay grietas diagonales en ambos sentidos.

El esfuerzo horizontal que origina la fisuración diagonal se calcula asimilándolo a la relación del esfuerzo a tracción de un disco cargado en su diámetro:

$$
\mathrm{V}_{\mathrm{D}}=\frac{\pi}{2} \mathrm{td}_{\mathrm{m}} \mathrm{f}_{\mathrm{tm}} \cos \theta
$$

donde $d_{m}$ es la longitud diagonal del paramento $\mathrm{y}_{\mathrm{tm}}$ es la resistencia a tracción de la fábrica de relleno.

Se observa fácilmente que no se producirá esta forma de rotura si la fábrica de confinamiento es de un material poco proclive al desplome, es decir, si tiene cierta armadura.

El cálculo de la estructura con obra de fábrica confinada puede hacerse sobre la base de un estado límite de servicio, presumiendo un factor de comportamiento (q) igual a 1 y comprobando la resistencia de la estructura contra las formas de colapso expuestas anteriormente.

En este caso la presencia de armaduras aumenta significativamente la resistencia de la estructura, al permitir desarrollar plenamente la resistencia diagonal de los paños de fábrica actuando como arriostramientos.

Si las fábricas de relleno tienen la armadura adecuada para mantener la integridad en los límites de deformabilidad puede considerarse un estado de esfuerzo máximo con un factor q más alto. En este caso puede esperarse que el modo de rotura diagonal degenere en una rotura de cizallamiento por rasante, donde la resistencia al deslizamiento del paramento se degrada a lo largo del ciclo del seísmo y, probablemente, se desarrolle una redistribución plástica de la respuesta del forjado. El estudio de la forma en que esto influye en el cálculo incluiría en análisis del pórtico de hormigón armado y se sale del objeto de este estudio.

(6) Abrams, D.P., and Paulson T.J., Measured nonlinear response of reinforced concrete masonry system, Proc. of the $5^{\text {th }}$ Canadian Masonry Symposium, Vancouver, 1989.

(1) CIB, International recommendations for design and erection of unreinforced and reinforced masonry structures, Publication No. 94, 1985.

(2) Commission of the European Community, Eurocode No. 8, Structures in Seismic regions, Brussels, 1989.

(3) Commission of the European Community, Eurocode No. 6, Common unified rules for masonry structures, Brussels, 1988.

(4) Commission of the European Community, Eurocode No. 6, Additional clauses, Common unified rules for reinforced masonry structures, Brussels, 1990.

(5) Tomazevic, M. and Weiss P., Seismic behaviour of masonry buildings: reinforced versus unreinforced masonry, Proc. of the $9^{\text {th }}$ I.B.M. a.C., Berlin, 1991.
7) Calvi, G.M., Macchi G., and Zanon P., Random cyclic behaviour of reinforced masonry under shear action, Proc. of the $7^{\text {th }}$ I.B.Ma.C., Melbourne, 1985.

(8) Priestley, M.J.N. and Calvi G.M., Towards a capacity-design assessment procedure for reinforced concrete frames, Earthquake Spectra, Vol. 7, No. 3, 1991.

(9) Building Construction under Seismic Conditions in the Balkan Region, Volume 3: Design and Construction of Stone and Brick-Masonry Buildings, UNDP/UNIDO Project RER/79/015, Vienna, 1984.

(10) Cantù, E. and Zanon P., Combined Cyclic Testing Procedures in Diagonal Compression on Hollow Clay Block Reinforced Masonry, $6^{\text {th }}$ International Brick Masonry Conference, Roma, Maggio 1982, pp. 1007-1020. 